Fecha de recepción: marzo 2021

Fecha de aceptación: abril 2021

Versión final: mayo 2021

\section{El proyecto de diseño, el presupuesto $y$ la virtualidad}

Pablo Guzmán Paredes ${ }^{(1)}$

Resumen: El avance de la implementación de la nueva malla curricular de la carrera de Diseño Gráfico de la Pontificia Universidad Católica del Ecuador impone nuevos retos en la planificación académica para cada uno de los niveles, mejoras continuas y tener la capacidad de adaptarse a temas coyunturales, como por ejemplo, lo sucedido con la pandemia ocasionada por el COVID-19, el confinamiento y las medidas adoptadas para evitar una mayor propagación del virus llevó a los docentes a replantear sus metodologías frente a la imperiosa necesidad de dictar las diferentes cátedras de manera virtual.

En ese contexto, la capacitación del docente para dictar clases universitarias en la virtualidad y arrancar con nuevas asignaturas, tal como lo plantea la nueva malla curricular de la carrera, permiten generar nuevas estrategias metodológicas, principalmente porque para quienes ejercemos la profesión del diseño el planteamiento de presupuestos ha sido siempre un escollo que ahora se aborda desde el contexto del proyecto de diseño y con el aporte de todas las asignaturas del sexto nivel de la carrera.

Palabras clave: Diseño gráfico - presupuestos - costos - proyectos - evaluación - enseñanza.

[Resúmenes en inglés y portugués en las páginas 84-85]

(1) Pablo Guzmán Paredes. Adscrito a la Pontificia Universidad Católica del Ecuador, en la Carrera de Diseño Gráfico de la Facultad de Arquitectura, Diseño y Artes. Diseñador y Magíster en Administración de Empresas. Docente PUCE. Código ORCID: 0000-00025825-0796

\title{
Desarrollo
}

"El diseñador vende soluciones; estas soluciones lo convierten en una empresa de servicios, y su servicio se mide en tiempo". (Piazza, 2017, p. 16)

Un aspecto muchas veces incomprendido dentro de la práctica profesional de los diseñadores gráficos es el de cómo calcular el presupuesto real de su trabajo, es decir, cuánto 
cobrar se ha vuelto, en muchos casos, un dolor de cabeza en la práctica profesional, dato que se ve evidenciado en la encuesta realizada por el área de seguimiento a graduados de la Carrera de Diseño Gráfico de la PUCE, en donde los antiguos alumnos de la carrera, graduados con la anterior malla curricular, manifiestan que el no saber cuánto cobrar es un problema recurrente en sus prácticas profesionales (Encuesta de retroalimentación). Muchos factores son los que, en nuestro medio, influyen para que se presente este fenómeno, pero, por experiencia del autor, este se da por la falta de preparación de los diseñadores en el área administrativa y de gestión empresarial, lo que deriva en el no saber cuánto se debe cobrar por trabajos de diseño y finalmente en problemas para mantener funcionando los negocios y emprendimientos de los diseñadores.

Otro aspecto importante a tener en cuenta es la actual situación económica que atraviesa el Ecuador (www.bce.fin.ec), ahondada por el estallido social de octubre 2019 y el confinamiento y estragos provocados por la pandemia en el año 2020, ante esto, se hace más evidente la necesidad de empezar a producir para ayudar a la economía de los hogares y según datos del GEM de la Espol, al año 2019 ocho de cada diez emprendimientos en el Ecuador fracasan en el primer año (GEM, 2019), una de las principales razones es que en un $90 \%$ de los casos el emprendedor no tiene conocimiento de metodologías que sean ágiles para aprender a controlar los procesos (emprendimiento.ec) y que seguramente, según el mismo portal, estos números se verán afectados aún más, de manera negativa, producto de la contracción económica provocada por la pandemia; este desconocimiento se ve evidenciado en la práctica diaria, los estudiantes de los diferentes niveles de la carrera, quienes empiezan a dar sus primeros pasos en el mundo laboral, suelen realizar consultas a los miembros de la planta docente sobre cuánto deben cobrar por determinado trabajo o elaboración de piezas gráficas. Lastimosamente este hecho es una muestra de una debilidad, que se había identificado y que se intenta corregir con el rediseño curricular de la carrera. Adicionalmente, según Piazza (2017) aproximadamente el $95 \%$ de diseñadores planean trabajar en su profesión de manera independiente, es decir que la gran mayoría piensa trabajar en el libre ejercicio profesional, en este punto, los futuros diseñadores deben entender que es importante conocer sobre diferentes conceptos administrativos, contables y de gestión, adicionalmente cobra suma importancia el conocimiento de que las políticas tributarias del Ecuador son bastante estructuradas, que es necesario obtener cualquiera de los dos tipos de registros de actividades comerciales y cualquier persona que ejerza una actividad comercial debe realizar periódicamente sus declaraciones impositivas de acuerdo a los diferentes regímenes que el país contempla (www.sri.gob.ec), además de la licencia o patente municipal que el cabildo del Distrito Metropolitano de Quito contempla para las actividades comerciales (www.quito.gob.ec).

Es de esta manera que, con el avance de la implementación del rediseño de la malla curricular en la carrera de Diseño Gráfico en la Pontificia Universidad del Ecuador (Rediseño Carrera de Diseño Gráfico PUCE, 2016) se presenta en el sexto nivel la asignatura de Análisis de costos y presupuestos la cual tiene como objetivo general conocer cómo se comportan los diferentes sistemas que constituyen un proyecto de diseño dentro de la práctica profesional (Resultados de aprendizaje Carrera de Diseño Gráfico PUCE, 2020). Dicha implementación presupone un reto, principalmente porque, como lo indica Del Vecchio (2015), las escuelas de diseño no abordan una gran cantidad de aspectos que de- 
ben conocerse en la práctica profesional. Es necesario fomentar en los futuros diseñadores el análisis global de sus proyectos, incluyendo temas importantes como lo son los temas de presupuestos económicos.

Partiendo de la premisa que el diseño es una disciplina proyectual (Mazzeo, 2017), la enseñanza del diseño en las escuelas no puede ni debe centrarse únicamente en el desarrollo del proceso creativo, Frascara (2017) indica que es necesario que la preparación de los futuros diseñadores debe ser desde y para la realidad, bajo esta aseveración, se plantea que es necesario reforzar el aprendizaje de los futuros profesionales del diseño con temas inherentes a la gestión profesional y no únicamente al diseño, propiamente dicho, esta preparación integral les permitirá, partir de la obtención de su título profesional, o incluso antes, empezar a desenvolverse de manera inmediata en el mercado laboral, por este motivo, el sexto nivel del rediseño de la carrera de Diseño Gráfico de la PUCE incluye la asignatura de Análisis de costos y presupuestos, dicha cátedra propone trabajar con los estudiantes en la configuración global de un proyecto, partiendo desde el análisis de datos que permitan formular el problema de diseño, pasando por el planteamiento de hojas de gestión que les permitan a los estudiantes realizar el respectivo monitoreo y evaluación de las diferentes fases del proyecto, también por el análisis del tipo de producto que plantearán como solución, de esta manera podrán tener una cantidad de elementos que permitirán finalizar el ciclo con la adecuada elaboración de una cotización, la cual deberá contar con valores reales del trabajo de cada uno de los grupos, dichos valores deberán contemplar el costo de la estructura de funcionamiento, para el ejercicio académico se presupone que los miembros del grupo de trabajo conforman una agencia de diseño, dicha estructura se compone, en términos financieros, de costos fijos y costos variables (Piazza, 2017) y el manejo del tiempo empleado en las diferentes etapas del proceso de diseño, de esta manera los estudiantes tienen un acercamiento real a la vida laboral y las distintas complejidades que se presentan para los diseñadores.

El principal reto que esta implementación plantea desde el primer nivel de la carrera, es el de trabajar con la metodología de aprendizaje basado en problemas (Escribano y Del Valle, 2010. p. 20), y su aplicación directa a proyectos de diseño, en esta metodología que propone abordar el problema de manera integral (Tobón, 210), los estudiantes deben entender todo el contexto relacionado con el problema y no resolver individualmente los aspectos que el este presenta, en este marco, los diferentes niveles de la carrera, dentro de su planificación para cada periodo académico, trabajan para vincular a las diferentes asignaturas que lo conforman para llegar a la consecución de proyectos de diseño abordados desde diferentes ámbitos. Asimismo, es importante mencionar que el mantenimiento de las medidas implantadas para detener el avance del COVID-19 en Ecuador ha obligado a las diferentes universidades, y en esta caso a la PUCE en particular, a que el sistema de clases virtuales se mantenga ya por varios periodos académicos (www.elcomercio.com), lo que ha derivado en que los docentes deban capacitarse en metodologías de enseñanza en ambientes virtuales, esto permite el uso de diferentes plataformas como soporte del proceso de enseñanza.

De esta manera, el planteamiento de la asignatura se desarrolla en conjunto con el proyecto de Taller de diseño para nuevos medios, correspondiente al $6^{\circ}$ nivel de la carrera, y conjuntamente con las asignaturas de Modelado y animación 3D y Edición de medios 
audiovisuales (Malla curricular Carrera de Diseño Gráfico PUCE). La asignatura de taller divide su planificación en dos proyectos, uno corto que tomará la mitad del periodo académico y otro largo que abarcará la totalidad del periodo, es en este proyecto en el que la asignatura de Análisis de costos y presupuestos trabaja, de esta manera, en la primera mitad del periodo la cátedra constituye en un apoyo para la etapa de investigación, los estudiantes deben realizar la recolección de información relacionada con el tema según los lineamientos entregados en la materia de Taller, el aporte se hace presente al empezar a realizar análisis de la información obtenida, en esta etapa los grupos de trabajo deben lograr realizar la formulación y planteamiento del problema, enfocando siempre el análisis hacia el diseño gráfico, es decir que los estudiantes deben identificar para posteriormente resolver un problema de comunicación visual, se realiza un primer planteamiento de los objetivos tanto general como específicos del proyecto y los resultados esperados del proyecto que ayudarán a cumplir dichos objetivos, en la materia de Taller se plantearán las estrategias que permitirán conseguir dichos resultados, esto posteriormente se verá reflejado en la calidad en el diseño (Frascara, 2017, p. 29).

Como paso posterior, cada grupo debe establecer las diferentes etapas del proyecto en un cronograma bien estructurado (Méndez Álvarez, 2013), este aspecto es sumamente importante, pues, permite a cada grupo además, de establecer las diferentes fases pro-yectuales, los responsables de cada una de estas fases, los recursos necesarios para su correspondiente ejecución, se especifica el planteamiento de sus tiempos de ejecución, esto servirá posteriormente para identificar este rubro en el presupuesto final de cada proyecto, finalmente se establecen las fechas de inicio y final de cada fase, soportados en la planificación realizada al arrancar el periodo académico por la planta docente del nivel. Con la definición del cronograma se realiza la identificación de la ruta crítica del mismo (Argüello y otros, 2018), se identifican las diferentes fases del proyecto que van a necesitar de más tiempo de ejecución para que en ellas se ponga mayor cuidado en el uso de recursos. Es importante en este punto empezar a trabajar con programas que originalmente han sido desconocidos para la mayoría de los estudiantes, por ejemplo Microsoft Project o aplicaciones similares que se encuentran en línea, este tipo de programas permiten elaborar de manera sistemática cronogramas, asignar tiempos, responsables y recursos, de igual manera, permiten elaborar el cálculo de la ruta crítica, es así que los estudiantes pueden entender fácilmente cómo elaborar el recurso e identificar su utilidad en el desarrollo del proyecto.

Al finalizar esta fase se cierra el primer cuarto de periodo académico, en donde se realiza el primer corte de evaluaciones y se puede identificar a los grupos o a los estudiantes que empiezan a presentar ciertos problemas con el desarrollo del proyecto, es importante realizar las intervenciones del caso para solucionar las dificultades presentadas y poder mantener al grupo nivelado en conocimientos.

La segunda unidad arranca con la identificación teórica del tipo de productos, desde el punto de vista del mercadeo (Lamb y otros, 2011. p. 337), para que los estudiantes conozcan la clasificación de los productos y logren identificar en qué categoría se colocaría su posterior objeto diseñado y qué tipo de atributos deberá tener, es importante indicar que hasta este momento la asignatura de Taller trabaja con mayor énfasis en el proyecto que dura solo la mitad del periodo académico (citar planificación del nivel). En esta unidad se establecen parámetros de competitividad de los productos, los estudiantes deben conocer sobre, a 
partir de los atributos planteados cómo su producto se volvería competitivo en el mercado, qué tipo de competencia podría tener, qué existe en el mercado, cómo destacarse frente a lo existente son parámetros que empiezan a dejar asentado para el posterior desarrollo del proyecto. A esta altura los estudiantes ya manejan diferentes conceptos administrativos y de gestión relacionados con el uso y optimización de recursos, humanos, físicos, económicos y otros que se encuentren dentro de la ejecución del proyecto (Caldas y otros, 2017).

Conocer de nuevos conceptos e interiorizarlos es parte de los aportes de la asignatura a la formación académica de futuros profesionales del diseño, partiendo del hecho de que, en su mayoría, son temas que no se habían profundizado en asignaturas anteriores de la carrera, es entonces importante que los estudiantes empiecen a familiarizarse con conceptos administrativos y financieros, temas que la experiencia docente indica que los estudiantes no los conocen y en algunos casos ni los han escuchado en ninguna ocasión, lo que consecuentemente que son temas complejos hasta llegar a entenderlos y profundizar en ellos. Es por este motivo que se hace especial énfasis, durante el desarrollo de la asignatura, en explicar, conversar y debatir sobre dichos conceptos, que al ser muy variados se aprovechan las diferentes situaciones coyunturales dentro de la realidad local, nacional, regional y mundial para explicar con ejemplos reales que resulta de gran ayuda didáctica para lograr una mejor comprensión por parte de los estudiantes. Utilizando diferentes métodos que lleven a un mejor entendimiento de cada uno de los temas que la asignatura plantea. Cada una de las fases de la asignatura debe ir de la mano del desarrollo del proyecto, por este motivo, al iniciar la segunda mitad del periodo académico, cuando la asignatura de Taller arranca ya con el desarrollo del producto, la cátedra de Análisis de costos y presupuestos pone énfasis en la elaboración del presupuesto económico del proyecto, en este punto es donde empiezan a aparecer las mayores complicaciones desde el punto de vista académico, la experiencia al impartir asignaturas de este tipo indica que existe un déficit de conocimiento del manejo de software como hojas de cálculo por ejemplo, programas como Microsoft Excel, herramienta poderosa pero que según la Ing. Nathalia Pérez, experta en la enseñanza del programa, en entrevista personal indica que: "el Excel es el programa del paquete Microsoft Office más complejo en aprender porque requiere lógica matemática y razonamiento lógico y hay una deficiencia desde la escuela en eso y por este motivo la gente le ve tan difícil”, es por este motivo que los ejercicios que se realizan en la asignatura no pretenden el uso profundo de la herramienta sino que lo que se pretende es que los estudiantes puedan realizar cálculos simples con los que logren plantear sus datos de costos y gastos y así elaborar una cotización. La aplicación de fórmulas en la herramienta es un problema que se subsana con la entrega por parte del docente de una plantilla con fórmulas elaboradas previamente que les permite a los estudiantes llenarla de acuerdo a los datos que cada proyecto presenta y así obtener el mencionado presupuesto. En este punto la temática aborda conceptos más abstractos para un diseñador, conocer y reconocer qué es un activo, un pasivo o el patrimonio es un tema complejo para los estudiantes, usualmente son conceptos que no han sido conocidos o utilizados ni en el transcurso de la carrera ni en su vida familiar, principalmente porque hasta este momento de su carrera universitaria no existen asignaturas relacionadas con la gestión, las finanzas y la administración de empresas. Es importante indicar que la intención de la cátedra no es convertir a los estudiantes en expertos en finanzas o administración, es simplemente dar 
una visión global de todos los factores que integran un proyecto, en este caso en particular un proyecto de diseño y que dichos factores les serán útiles para llegar a la elaboración adecuada del presupuesto del mismo y a mantener un control óptimo de recursos, no solo económicos, sino físicos, humanos y de otros tipos, para su desarrollo.

Es sumamente importante que los estudiantes aprendan a diferenciar entre gastos y costos, costos fijos y variables, a calcular la rentabilidad y el punto de equilibrio y que conozcan la normativa ecuatoriana para la declaración de impuestos para profesionales en el libre ejercicio y microempresarios, factores que en la práctica profesional adquieren importancia, dado que en el Ecuador, como se lo había indicado en líneas anteriores, existe un rígido control tributario, los estudiantes deben adquirir conocimiento sobre registro único de contribuyentes, régimen simplificado, declaraciones de impuestos al valor agregado y a la renta, régimen de microempresas, retenciones, entre otros; estos conceptos son útiles para conocer sobre el manejo adecuado de los recursos económicos. No se debe profundizar en cálculos financieros avanzados, se pretende que los estudiantes conozcan los conceptos básicos y la lógica de su aplicación para que al momento de ejercer profesionalmente puedan realizar el monitoreo adecuado de los movimientos de sus negocios. Es de esta manera que el proceso académico finaliza con la elaboración de una cotización real del trabajo efectuado durante todo el periodo de estudios y con el proyecto planteado desde el inicio por la materia de Taller, para el cierre del ciclo, los diferentes grupos de trabajo han realizado todo el desarrollo de diseño en donde se deben identificar los tiempos de trabajo que se los ha dividido en tiempos de gestión, que comprende la investigación y la validación, trabajo de diseño propiamente dicho y trabajo operativo (Piazza, 2017), esta diferenciación permite identificar plenamente los tiempos de trabajo en cada etapa para después calcular el costo final del proyecto, adicionalmente, con base en el trabajo anterior de algunos docentes de la carrera, se ha elaborado una tabla de cálculo de valores, dicha tabla contiene un desglose de gastos personales del estudiante, en este caso del grupo de trabajo, detalla los valores mínimos que necesita para su manutención, este valor se lo toma como base para la obtención de recursos económicos mensuales, adicionalmente a esto, la tabla mencionada tiene campos en diferentes rubros de costos fijos y variables de la estructura de trabajo, equipos, suministros, mano de obra, servicios básicos y otros valores son contemplados, automáticamente, esto se lo realiza, como se lo indicó anteriormente, mediante una tabla que se encuentra con fórmulas ya elaboradas previamente para que los estudiantes se limiten a llenar los datos según cada proyecto, dicha tabla calcula automáticamente las depreciaciones de los equipos y conjuntamente con los otros costos inmersos en el proyecto, los prorratea de acuerdo a las horas de trabajo que inicialmente se identificaron y registraron. Finalmente las fórmulas llevan a la obtención de un valor neto que es el total del costo del trabajo más la rentabilidad que debe percibir quien ofrece el servicio, en este caso, el diseñador; de ser el caso de trabajar con un cliente final, este valor final se debe ser presentado para su respectivo análisis. La tabla, al ser alimentada con la información de gastos y costos, calcula automáticamente el punto de equilibro del proyecto $\mathrm{y}$, como se indicó, la rentabilidad, por lo tanto se asegura que de no darse la aceptación de la misma, el diseñador tendrá un margen para negociar con el cliente, en este caso, al ser resultado de un proceso académico, la tabla calcula el costo del proyecto con la rentabilidad deseada. Es necesario indicar que esta fórmula de elaboración de cotizaciones no es una metodo- 
logía única aplicada para este fin, pero sí reúne conceptos administrativos y ha sido aplicada en la práctica por el autor del presente artículo, docente de la asignatura, lo que ha permitido mantener en orden los flujos del negocio.

\section{Estrategias utilizadas}

Una particularidad que se debe tener en cuenta dentro de los retos que presenta la virtualidad, modalidad en la que hasta el momento se ha desarrollado la cátedra, es que las aulas virtuales se han convertido en una herramienta esencial en el desarrollo de la enseñanza, y trabajar en la enseñanza del diseño desde la virtualidad es un reto mucho más grande, partiendo del hecho de que el uso que tradicionalmente que se ha dado a las aulas virtuales es el de repositorios digitales de información que permite a los estudiantes acceder al material de la asignatura, en un estudio realizado por Moreira y compañía (2018) se indica en sus resultados que tanto docentes como estudiantes enfatizan en que el aula virtual es utilizada para la subida de archivos, es decir que no es aprovechada como una verdadera herramienta de enseñanza y lastimosamente no se utilizan recursos de comunicación e incluso las diferentes opciones para evaluación son poco utilizadas; la capacidad de aprovechar el potencial que tiene el aula virtual para que sea una herramienta de trabajo depende del docente, incentivar al trabajo colaborativo para que el nivel de compromiso y satisfacción por parte de los estudiantes sea mayor permitirá obtener mejores resultados de aprendizaje (Navarro Soria y otros, 2019). De esta manera el aula virtual pasa de convertirse de un simple repositorio de información a una potente herramienta para el correcto desempeño de estudiantes y docentes. Es ahí donde radica la diferencia entre repositorios y aulas virtuales, con un aula bien planificada es posible realizar mayor cantidad de actividades que permitan que la virtualidad sea atractiva para los estudiantes, por lo tanto en la asignatura de Análisis de costos y presupuestos se pretende el permanente uso del aula virtual como herramienta de alimentación de información, se plantean foros de discusión de temas coyunturales, se elaboran documentos colaborativos en donde se analiza la situación del diseño desde el punto de vista del emprendimiento, el principal objetivo de este tipo de ejercicios es alimentar en los estudiantes una cultura de análisis y crítica constructiva de las diversas situaciones que intervienen en el ambiente en el que se desarrollan, tanto familiarmente como a nivel profesional, esto, tomando en cuenta que se encuentran a poco menos de un año de finalizar sus estudios de pregrado y que se trata con alumnos adultos, por lo que el nivel de análisis debe ser alto tanto en profundidad como en calidad.

El constante avance de las tecnologías de la comunicación (TICs) ha traído un nuevo reto a la enseñanza y lógicamente a la andragogía, utilizar de manera adecuada estas herramientas presupone un esfuerzo mayor tanto para docentes como para estudiantes, quienes están más acostumbrados al uso de aplicaciones, como por ejemplo las redes sociales, como instrumentos de conexión social, pero estas mismas redes pueden convertirse en herramientas para difundir conocimiento y generar estrategias de trabajo colaborativo (Marín-Díaz y Cabero-Almenara, 2019). Los cambios que este reto presuponen se han visto acelerados por la situación actual, el confinamiento obligó a que los docentes anali- 
cen las mejores opciones de trabajo colaborativo para sobrellevar de mejor manera cada una de las asignaturas.

Las herramientas de trabajo colaborativo, como por ejemplo las Wikis o plataformas como Padlet, Stormboard y otros, presentan entre sus beneficios su fácil manejo (Vitalaru, 2019), estas herramientas en general, permiten que los diferentes participantes aporten con sus ideas, conocimientos e investigaciones al desarrollo de un producto final de calidad y principalmente que ayude en el cumplimiento de los objetivos planteados desde las diferentes asignaturas a la carrera. Es de esta manera que con el desarrollo de la asignatura se pretende que los estudiantes interioricen los diferentes conceptos con la ayuda de diversas herramientas de trabajo colaborativo y plataformas virtuales que ayudarán a realizar tareas sincrónicas y asincrónicas entre los diferentes grupos de trabajo, permitirán fomentar el análisis y generar el debate de estos temas que resultan importantes dentro del desarrollo de la profesión. Se puede afirmar que aprovechar correctamente las TICs como herramientas de trabajo colaborativo puede ser sumamente útil dentro de la planificación de cada asignatura y ayudarán al cumplimiento de los objetivos planteados, especialmente en el ámbito del diseño donde la interacción entre todos los actores que intervienen en el proceso de aprendizaje es fundamental.

Los avances tecnológicos propios de nuestra era han llevado a los diferentes sistemas de educación a adaptarse rápidamente a las tendencias y a romper paradigmas que hacían que la educación tradicional se impongan, uno de esos cambios es la pedagogía inversa, es importante, al adoptar esta metodología, tomar en cuenta que se debe encontrar el adecuado equilibrio entre lo tradicional y lo virtual como lo explica Calatrava (2019), quienes realizan labores docentes están en la obligación de planificar adecuadamente cada una de las asignaturas para obtener los mejores resultados explotando los diversos recursos tecnológicos que se encuentren al alcance, siempre tomando en cuenta que no es beneficioso saturar a los estudiantes de labores, por ese motivo, en la escuela de Diseño Gráfico de la PUCE se trabaja cada nivel por proyectos prácticos (Frascara, 2017, pp. 33) en el que todas las materias colaboran en conjunto para realizar un aporte significativo en el desarrollo del mismo y así las tareas que las asignaturas envían a sus estudiantes están destinadas a resolver los diferentes aspectos del proyecto de Taller.

Adicionalmente, la metodología de aula inversa tiene como ventajas el trabajo personalizado con los estudiantes, que se puede incrementar el compromiso del alumnado, que cada uno trabaje a su propio ritmo, el uso de TICs; pero también presenta desventajas como la falta de recursos de ciertos estudiantes para acceder a una conexión adecuada a Internet o a equipos de computación útiles, que deben realizar un trabajo previo de lec-tura e investigación antes de iniciar un módulo nuevo, que el profesor deberá dedicarle más tiempo a la preparación de sus temas, la falta de relación personal dado que el medio de comunicación es una pantalla, que no todos los estudiantes tienen la misma capacidad para aprender de forma autónoma (Berenguer-Albaladejo, 2016); de todas maneras, estos puntos en contra pueden ser resueltos con el componente tradicional de la metodología, en la personalización de las clases donde se encontrarán docente y alumnos es cuando se podrán resolver dudas e inquietudes que el trabajo autónomo genere y así obtener mejores resultados.

Es por este motivo que la metodología del aula inversa es utilizada en ciertos aspectos para romper con la rutina habitual de las clases virtuales, se plantea que los estudiantes desarro- 
llen temas relacionados con la gestión y la administración para que los expliquen ante el resto de la clase, de esta manera se incentiva el análisis y debate, anteriormente mencionados, de los temas coyunturales que alimentan el conocimiento de los estudiantes.

\section{Problemas encontrados}

Uno de los problemas recurrentes que se reflejan en esta etapa de enseñanza virtualizada es conseguir la participación constante de los estudiantes en el desarrollo académico, mantener el interés en las horas de clase es otro reto importante, por eso se plantean las diferentes estrategias planteadas en líneas anteriores

En primer lugar es necesario señalar que es sumamente importante demostrar el compromiso que tiene el docente con su grupo y con los temas a tratar, la responsabilidad que cada docente tiene debe verse reflejada en el armado tanto de planificación como del aula virtual y en la presentación de cada uno de los temas de la asignatura, los estudiantes deben evidenciar que todos los profesores del nivel se encuentran en sintonía con el proyecto y el avance del mismo, de esta manera se demuestra el trabajo que existe desde las semanas previas al arranque del periodo académico.

Otro factor de mucha importante es el de mantener una adecuada interacción con los estudiantes (García, Serrano y otros, 2018), esto permite que ellos se sientan involucrados en el desarrollo de las actividades y su motivación aumente.

Finalmente, el mantener una comunicación activa por distintos medios, no solo el aula virtual o correo institucional, las plataformas de mensajería permiten estar en mayor contacto con los estudiantes y así fomentar la confianza basada en el respeto mutuo, este contacto se ve fortalecido a partir de la situación actual, puesto que según el estudio de Villora Matheus y Hamburger (2019) las herramientas sincrónicas como videoconferencias y grupos de chat eran usados medianamente por parte de los docentes, dadas las actuales circunstancias, estos medios de comunicación sincrónica se han tornado muy importantes dentro de la educación y, como se lo mencionó, ayudan a mantener la comunicación, siempre enmarcada en el respeto mutuo.

En cuanto a las ausencias repetidas de los estudiantes, en primer lugar se debe tomar en cuenta que un estudiante que se ausente de manera prolongada a sus clases debe estar atravesando alguna situación inusual, según Suárez Rivera y otros (2016), en nuestra sociedad las deserciones van relacionadas a diferentes factores, entre ellos académicos, personales y socioeconómicos. Estos factores inciden directamente en el rendimiento académico de los estudiantes. Por lo tanto se torna sumamente importante el evaluar individualmente cada caso, más aun en la situación actual, se deben considerar que adicionalmente a los factores mencionados en época de pandemia incide plenamente el factor salud, familiares infectados con COVID-19 o incluso el mismo estudiante puede estarlo, asimismo, el estrés ante el futuro económico incierto dada la situación que atraviesan nuestros países es otro factor que afecta directamente a la población joven (Velázquez Hernández, 2020) y es muy probable que esto lleve a un estudiante a desertar o a ausentarse de sus clases. 
Es importante el análisis personalizado de cada caso, si el estudiante realmente atraviesa una situación con la que no puede lidiar, es deber del docente reportar el caso oportunamente para que desde coordinación de carrera se derive a los estamentos universitarios pertinentes para que estos brinden el apoyo necesario y así se pueda controlar de alguna u otra manera su situación personal, Fuertes Seder y otros (2018) mencionan que el uso de nuevas metodologías de estudio son útiles en mantener la motivación de los estudiantes, pero es más importante el trabajo en conjunto entre los diferentes organismos de la institución para ayudar a los estudiantes, existen situaciones en las que los docentes no estamos en la capacidad de manejarlas y son los departamentos especializados los llamados a atenderlas, es responsabilidad del docente detectar estas situaciones para reportarlas oportunamente. De esta manera se propende a mantener un saludable ambiente entre el grupo de trabajo, la empatía tanto del docente hacia los alumnos como viceversa es un factor sumamente importante que debe manejarse dentro de la enseñanza.

Dentro del desarrollo propio de la cátedra el principal problema encontrado es, como se lo había mencionado en líneas anteriores, que los estudiantes no tienen conocimiento de los conceptos tratados, términos administrativos o financieros les son sumamente desconocidos, y la primera experiencia al impartir la asignatura evidenció que algunos términos se prestan para ser confundidos, especialmente aquellos términos que manejan conceptos similares, como por ejemplo activo y pasivo corriente, los estudiantes presentan cierta complicación en la comprensión de conceptos de este estilo.

Dentro de la fase investigativa, la coyuntura de los proyectos ha llevado que ésta no se presente fortalecida con datos concluyentes, por lo que la formulación del problema no puede ser sustentada correctamente, hecho que es corregido en la asignatura de Taller para darle mayor sustento a la información y que a partir de los correctos planteamiento y formulación del problema lleguen a tomarse acertadas decisiones de diseño. Esto repercute también en el frágil planteamiento de objetivos y resultados esperados.

Asimismo, como se mencionó anteriormente, no es usual en los estudiantes el uso de herramientas tecnológicas que no están relacionadas con el diseño por lo que se les complica su manejo, al momento del cierre del presente artículo, tanto el primer grupo que recibió la asignatura como el que actualmente se encuentra cursándola no han mostrado mayor problema en el uso de herramientas como Microsoft Project, herramienta que les ha resultado relativamente fácil su comprensión, uso y aplicación, lo que aporta sustancialmente al entendimiento del concepto asociado; por otro lado, el programa Microsoft Excel sí presenta mayores complejidades, su conocimiento y manejo son muy básicos por lo que se les dificulta la elaboración de tablas y fórmulas, esta dificultad se ve solventada con el uso de una tabla pre fabricada que, como fue mencionado, permite a los estudiantes cargar la información de costos y gastos del proyecto y así obtener la cotización final del producto de diseño. Resulta importante el fortalecimiento en el aprendizaje de herramientas de este tipo.

Otro factor que presenta complicaciones dentro del proceso de aprendizaje es el del entendimiento del régimen impositivo, al ser el Ecuador un país que cuenta con una cultura de impuestos, es sumamente común la emisión de facturas y a nivel empresarial no se realizan transacciones comerciales con terceros sin la presentación de una factura a la entrega del producto, ya sea físico o un servicio, como es el caso del diseño, por lo tanto 
es importante que los estudiantes conozcan las posibilidades que tienen para entrar en el mundo laboral con la oferta de sus servicios, algunos estudiantes ya tienen su registro único de contribuyentes pero no tienen conocimiento de cuáles son sus obligaciones o del llenado de las respectivas declaraciones, sistema que el Servicio de Rentas Internas del Ecuador ha ido desarrollando con los años y simplificando sus procedimientos, por lo que en la actualidad resulta muy sencillo el llenar correctamente dichas declaraciones y que estas sean presentadas a tiempo, eliminando así la posibilidad de acarrear multas por incumplir con estos deberes del contribuyente.

\section{Conclusiones}

Se concluye que el Ecuador es un país en donde el emprendimiento tiene un amplio espacio en el mercado pero que por el desconocimiento de herramientas y procedimientos en el manejo de la gestión del negocio tiende a fracasar en un altísimo porcentaje en el primer año de operaciones; la actual situación económica del Ecuador obliga a los estudiantes a empezar a producir para colaborar con la economía de sus respectivos hogares, por lo tanto es sumamente importante que adquieran conocimientos de temas administrativos y financieros para que esta temprana inclusión en el mercado laboral no se vea afectada por factores ajenos al diseño propiamente dicho, pero que pueden llevar a obtener un sentimiento de frustración que puede llevar al estudiante a tener diferentes sensaciones adversas sobre su profesión, de igual manera sucede con los profesionales ya egresados, con la antigua malla de la carrera en la que se topaban estos temas sin mucha profundidad y orden, no pudieron obtener la información adecuada para incursionar en el mundo del emprendimiento o en la oferta de servicios de manera adecuada.

Al ser el diseño una carrera en la que sus profesionales en un alto porcentaje tienden al libre ejercicio profesional, es imperiosamente necesaria la preparación en estos temas de gestión, administrativos, contables y financieros, sin propender a la especialización en estos temas, el conocimiento básico de cómo funciona una empresa se torna fundamental para el ejercicio independiente de la profesión, si bien es cierto que en la práctica lo más aconsejable es contratar los servicios de personas que tengan conocimientos y que se hayan preparado en estos temas, no está por demás que los diseñadores los conozcan para que puedan entender sobre el manejo de sus finanzas y así evitarse problemas posteriores. La situación de la virtualidad ha llevado a los docentes a ser más ingeniosos en el manejo de sus diferentes cátedras, por lo que se ha fortalecido el uso de aulas virtuales, las cuales deben dejar de ser repositorios de información y deben convertirse en elementos de diseño que aporten al proceso de aprendizaje en donde se desarrollen actividades de diferente tipo como trabajo colaborativo, foros, discusiones, aulas inversas y demás, de esta manera se pretende mantener el interés de los estudiantes y así procurar que el proceso de la asignatura fluya adecuadamente.

Finalmente, es importante poner especial atención en casos de estudiantes que puedan presentar problemas, detectarlos a tiempo para derivarlos a los organismos universitarios correspondientes y así procurar un correcto desenvolvimiento de la asignatura. 


\section{Recomendaciones}

Como recomendaciones del presente artículo se puede mencionar que es importante desde los primeros niveles de universidad fortalecer el conocimiento de diferentes aspectos de la realidad mundial, regional, nacional y local, este aspecto ayudará a los estudiantes a tener un conocimiento y un entendimiento de su entorno, saber qué sucede a su alrededor es un factor importante en el desarrollo de cualquier proceso de diseño, diseñar para la gente. Este refuerzo ayudará también a fortalecer los procesos de investigación para que los resultados sean acorde a la realidad del proyecto, conocer el estado de la economía, la situación de la industria, del mercado, del consumidor ayudarán a tomar acertadas decisiones de diseño que se enmarquen en la realidad del usuario y así resolver de mejor manera el problema de diseño.

\section{Bibliografía}

Area, M., San Nicolás, B., y Sanabria, A. (2018). Las aulas virtuales en la docencia de una universidad presencial: la visión del alumnado. RIED. Revista Iberoamericana de Educación a Distancia, 21(2), pp. 179-198. doi: http://dx.doi.

Org/10.5944/ried.21.2.20666

Argüelo, E., Villagrán W., Buenaño L., Altamirano, M. y Cruz, E. (2018). Modelación matemática para la estimación del tiempo de la ruta crítica de un proyecto utilizando el método PERT. Polo del Conocimiento. Vol 3, No 11, p.176-188. DOI: 10.23857/pc.v3i1.786

Calatrava, V. Transformación digital en la educación: blended learning y flipped class-room o aula invertida. Recuperado el 11 de agosto de 2020 de: https://www.d2l.com/es/blog/ transformacion-digital-en-la-educacion-blended-learning-y-flipped-classroom-o-aulainvertida/

Caldas, M., Carrión, R., y Heras, A. (2017). Gestión administrativa (Empresa e iniciativa emprendedora). Madrid. Editex.

Carrera de Diseño Gráfico PUCE. Malla de la Carrera de Diseño Gráfico PUCE. Recuperado el 11 de abril de 2021 de: https://puceeduec-my.sharepoint.com/:f:/g/personal/pxguzman puce_edu_ec/EvlWM1I8ycBPpz294MbZlDwBfaha-CEnDniLBKA_0TiZ3Q?e=mQyTpT

Carrera de Diseño Gráfico PUCE. Recuperado el 10 de abril de: https://www.puce.edu.ec/ wp-content/uploads/2021/03/Folleto_Disen\%CC\%83o-Grafico.pdf

Del Vecchio, F. (2015). Diálogos con diseñadores. Tigre. Argentina.

Encuesta de retroalimentación al proceso formativo de los años 2018 y 2019. Recuperado el 11 de abril de 2021 de: https://docs.google.com/forms/d/e/1FAIpQLSdSzRb4Ed8SC u4c4NX1Y5hWl5dzOnMPOkOsTf7SK-Xqw8FAZQ/closedform

Escribano, A. Del Valle, A. (Coords.). (2010). El aprendizaje basado en problemas (ABP). Madrid: Narcea S.A. Ediciones.

Frascara, J. (2017). Enseñando diseño. Buenos Aires: Infinito. 
Fuertes Seder, A., Ferrís Castell, R., \& Grimaldo Moreno, F. (2018). ¿Un cambio de metología que aumente la satisfacción y motivación del estudiante favorece su aprendizaje? Experiencias en el aula. Actas de las Jenui, vol3. pp. 335-342

García, B., Serrano, E. L., Ponce Ceballos, S., Cisneros-Cohernour, E. J., Cordero Arroyo, G., y Espinosa Díaz, Y. (2018). Las competencias docentes en entornos virtuales: un modelo para su evaluación. RIED. Revista Iberoamericana de Educación a Distancia, 21(1), pp. 343-365. doi: http://dx.doi.org/10.5944/ ried.21.1.18816

Global Entreprneurship Monitor Ecuador 2019. Recuperado el 8 de abril de 2021 de: https:// www.espae.espol.edu.ec/wp-content/uploads/documentos/GEM_Ecuador_2019.pdf. Recuperado el 8 de abril de 2021.

Información económica del Banco Central del Ecuador. Recuperado: el 3 de abril de 2021: https://www.bce.fin.ec/index.php/informacioneconomica

Lamb, C., Hair, J., y McDaniel, C. (2011). Marketing. (11 ed.) México DF. CENGAGE Learning.

Marín-Diaz, V., y Cabero-Almenara, J. (2019). Las redes sociales en educación: desde la innovación a la investigación educativa? RIED. Revista Iberoamericana de Educación a Distancia, 22 (2), pp. 25-33. doi: http://dx.doi.org/10.5944/ ried.22.2.24248

Mazzeo, C. (2017) Diseño y sistema. Bajo la punta del iceberg. Ciudad Autónoma de Buenos Aires: Infinito.

Mendez Alvarez, C. E. (2020). Metodología de la investigación: Diseño y desarrollo del proceso de investigación en ciencias empresariales. (5 ed.) Alfaomega Colombiana S.A.

Navarro Soria, Ignasi; González Gómez, Carlota; López Monsalve, Begoña; Contreras Fontanillo, Aurora. "Aprendizaje cooperativo basado en proyectos y entornos virtuales para la formación de futuros maestros». Educar, [en línea], 2019, Vol. 55, n. 2, pp. 519-41, https://www.raco.cat/index.php/Educar/article/view/v55-n2-navarro-gonzalez-lopezcontreras [Consulta: 21-07-2020].

Piazza, J. (2017). Cómo presupuestar diseño. Buenos Aires: Platina \& Shablon. . El diseño como negocio. Buenos Aires: Platina \& Shablon.

¿Por qué fracasan los emprendimientos? Recuperado el 8 de abril de 2021 de: https:// emprendimiento.ec/

Preguntas frecuentes - patente. Recuperado el 8 de abril de: https://www.quito.gob.ec/index. $\mathrm{php} /$ municipio/270-preguntas-frecuentes-patente

Régimen impositivo para microempresas. Servicio de Rentas Internas. Recuperado el 4 de abril de 2021 de: https://www.sri.gob.ec/regimen-impositivo-para-microempresas

Suarez Rivera, D., Gómez Bandera, C., \& Pulido Pulido, Y. (2016). Estudio, medición y reporte de las causas asociadas a la deserción, una experiencia significativa para la institución. Congresos CLABES. https://revistas.utp.ac.pa/index.php/clabes/article/view/1025

Tobón Tobón, S. (2010) Formación integral y competencias. Pensamiento complejo, currículo, didáctica y evaluación. Bogotá: Ecoe Ediciones.

Tortosa Ybáñez, María Teresa; Grau Company, Salvador; Álvarez Teruel, José Daniel (coords.). XIV Jornades de Xarxes d'Investigació en Docència Universitària. Investigació, innovació i ensenyament universitari: enfocaments pluridisciplinaris = XIV Jornadas de 
Redes de Investigación en Docencia Universitaria. Investigación, innovación y enseñanza universitaria: enfoques pluridisciplinares. Alacant: Universitat d'Alacant, Institut de Ciències de l'Educació, 2016. ISBN 978-84-608-7976-3, pp. 1466-1480

Velázquez Hernández, J. (2020). Impacto de la pandemia SARS-COV2 en nuestros jóvenes. Revista Latinoamericana de Investigación Social, 3(1), 9-14. Recuperado a partir de http://revistasinvestigacion.lasalle.mx/index.php/relais/article/view/2633 HYPERLINK "https://revistas.utp.ac.pa/index.php/clabes/article/view/1025"

Villoria Matheus, H., Hamburger, J. (2019). Uso de las herramientas comunicativas en los entornos virtuales de aprendizaje. Chasqui. Revista Latinoamericana de Comunicación, N. ${ }^{\circ}$ 140, abril - julio 2019 (Sección Informe, pp. 367-384)

Vitalaru, B. (2019). Wikis como herramientas de trabajo colaborativo complementario y desarrollo de recursos para la formación en traducción a nivel universitario: Análisis retrospectivo. Revista de Educación a Distancia (RED), 19(59). https://doi.org/10.6018/ $\mathrm{red} / 59 / 02$

¿Volverán a clases presenciales las universidades particulares? Recuperado el 10 de abril de 2021 de: https://www.elcomercio.com/actualidad/clases-presenciales-universidadesparticulares-quito.html

\begin{abstract}
The progress of the implementation of the new curriculum for the Graphic Design career at the Pontificia Universidad Católica del Ecuador imposes new challenges in academic planning for each of the levels, continuous improvements and having the ability to adapt to current issues, such as, for example, the situation with the pandemic caused by COVID-19, the confinement and the measures adopted to prevent a further spread of the virus led teachers to rethink their methodologies in the face of the urgent need to teach the different chairs virtually.

In this context, the training of the teacher to teach university classes in virtuality and start with new subjects, as proposed by the new curriculum of the career, allows the generation of new methodological strategies, mainly because for those of us who practice the design profession the approach to the budget has always been a stumbling block that is now being addressed from the context of the design project and with the contribution of all the subjects of the sixth level of the career.
\end{abstract}

Keywords: Graphic design - budgets - costs - projects - evaluation - teaching.

Resumo: O avanço da implantação do novo currículo para a carreira de Design Gráfico na Pontifícia Universidade Católica do Equador impõe novos desafios no planejamento acadêmico para cada um dos níveis, melhorias contínuas e capacidade de adaptação às questões atuais, como Por exemplo, o que aconteceu com a pandemia provocada pela COVID-19, o confinamento e as medidas adotadas para evitar uma nova disseminação do vírus levaram os professores a repensar suas metodologias diante da necessidade urgente de ensinar virtualmente as diferentes cadeiras. 
Nesse contexto, a formação do professor para ministrar aulas universitárias na virtualidade e iniciar com novas disciplinas, conforme proposto pelo novo currículo da carreira, permite a geração de novas estratégias metodológicas, principalmente porque para nós que exercemos a profissão de design a abordagem $\mathrm{O}$ orçamento sempre foi um obstáculo que agora está sendo abordado a partir do contexto do projeto de design e com a contribuição de todas as disciplinas do sexto nível da carreira.

Palavras-chave: Design gráfico - orçamentos - custos - projetos - avaliação - ensino.

[Las traducciones de los abstracts fueron supervisadas por el autor de cada artículo] 